\title{
On the metal abundance in the core of M 87
}

\author{
S. Molendi ${ }^{1}$ and F. Gastaldello ${ }^{1,2}$ \\ 1 Istituto di Fisica Cosmica, CNR, via Bassini 15, 20133 Milano, Italy \\ 2 Università di Milano Bicocca, Dip. di Fisica, P.za della Scienza 320133 Milano, Italy
}

Received 11 May 2001 / Accepted 19 June 2001

\begin{abstract}
We revisit the XMM-Newton observation of M 87 to make a new and more detailed measurement of the metal abundance profile. After having verified that spectral fits with single temperature models show a dramatic abundance drop within 1 arcmin of the cluster core, we show that more appropriate models, which include a multi-temperature component to account for the strong temperature gradient observed in $\mathrm{M} 87$, and a power-law component to account for the emission of the nucleus and knot A, give a substantially flat abundance profile. The drastic abundance decrement found by fitting a single temperature component to the data is an artifact known as "Fe-bias" (Buote 2000a,b) following from the application of an oversimplified spectral model to the data.
\end{abstract}

Key words. X-rays: galaxies - galaxies: clusters - galaxies: individual: M 87

\section{Introduction}

Our proximity to the Virgo cluster allows us to study its core on physical scales unachievable in other similar systems. M 87, the giant elliptical galaxy at the center of Virgo, is not surprisingly amongst the most studied extragalactic objects in the sky. At X-ray wavelengths it has been observed by all major observatories. Deprojection of the X-ray images obtained with the IPC instrument on Einstein (Fabian et al. 1984) showed that the gas in M 87 has cooling times shorter than the Hubble time. Further evidence of cooling gas has come from the spectrometers on-board Einstein, which showed that the gas in M 87 was not single-temperature (e.g. Canizares et al. 1982). Subsequent observations with ROSAT (Nulsen \& Böhringer 1995), ASCA (Matsumoto et al. 1996) and BeppoSAX (Guainazzi \& Molendi 2000), showed evidence of a temperature decrement with decreasing radius. The ASCA and BeppoSAX data also showed evidence of a metal abundance increase towards the center of M 87 on scales of a few arcmin, which correspond to a few tens of kpc.

A first set of results from the XMM-Newton observation of M 87 have been recently published in Böhringer et al. (2001) and Belsole et al. (2001). One of the most striking results presented is a very marked drop in the abundance profiles of $\mathrm{Si}$ and $\mathrm{Fe}$ within 1 arcmin of the center of M 87. Böhringer et al. (2001) suggest that resonant scattering may be responsible for this drop. Mathews

Send offprint requests to: S. Molendi,

e-mail: silvano@ifctr.mi.cnr.it et al. (2001), using detailed radiation transfer calculations, remark that the resonant scattering effect is insufficient to explain the observed profile and that some sort of continuous opacity is required.

In a recent paper (Molendi \& Pizzolato 2001) we have shown that the multi-phase appearance of the EPIC spectra of M 87 within 2 arcmin from the core is not ascribable to the presence of a truly multi-phase gas but is rather the consequence of having, within the same line of sight, emission from gas at different radii with different temperatures. In this Letter we revisit the XMM-Newton observation of M 87; our goal is to remeasure the metal abundance profile by performing a more advanced spectral analysis of the EPIC data with models which take into account the projection effects leading to the multi-phase appearance of the gas in M 87.

The remainder of this letter is organized as follows. In Sect. 2 we describe the data preparation. In Sect. 3 we discuss the spectral models. In Sect. 4 we present and discuss the results of our analysis.

\section{Observations and data preparation}

We use XMM-Newton EPIC data from the PV observation of M 87/Virgo. Details on the observation, as well as results from a first analysis of this object, have already been published in Böhringer et al. (2001) and Belsole et al. (2001).

We have obtained calibrated event files for the MOS1, MOS2 and PN cameras with SASv5.0. Data were manually screened to remove any remaining bright pixels or 
hot column; for the PN camera we also exclude regions contaminated by out of time events. Periods in which the background is increased by soft-proton flares have been excluded using an intensity filter; we rejected all events accumulated when the count rate exceeds $15 \mathrm{cts} / 100 \mathrm{~s}$ in the $[10-12] \mathrm{keV}$ band for the MOS cameras and 25 for the PN. We have accumulated spectra in 4 concentric annular regions centered on the emission peak. We extend our analysis out to 3 arcmin from the emission peak. The bounding radii for the regions are: $0^{\prime \prime}$ and $30^{\prime \prime}$ for region $1 ; 30^{\prime \prime}$ and $1^{\prime}$ for region $2 ; 1^{\prime}$ and $2^{\prime}$ for region 3 and $2^{\prime}$ and $3^{\prime}$ for region 4 . We have removed point sources, and the substructures which are clearly visible from the X-ray image (e.g. Belsole et al. 2001) except in the innermost region. Here we have kept the nucleus and knot $\mathrm{A}$, the reason being that on angular scales this small it is not possible to exclude completely their emission. As discussed in Sect. 3 we prefer to fit the spectrum of this region with a model which includes a power-law component to fit the two point-like sources; note that we include only one power-law component because the two sources have similar spectra (Böhringer et al. 2001).

Spectra have been accumulated for MOS1 and MOS2 independently. The Lockman hole observations have been used for the background. Background spectra have been accumulated from the same detector regions as the source spectra. The line of sight galactic absorbing column depth towards the background field is somewhat smaller, $N_{\mathrm{H}} \sim$ $6 \times 10^{19} \mathrm{~cm}^{-2}$, than that found on the line of sight of M 87 . This has a negligible impact on the soft part of our M 87 spectra because the $N_{\mathrm{H}}$ variation will lead to a maximum difference of $10 \%$ in the background intensity, which is in turn never more than $2 \%$ of the source intensity in the $0.5-1.0 \mathrm{keV}$ energy range.

The vignetting correction has been applied to the spectra rather than to the effective areas, similarly to what has been done by other authors who have analyzed EPIC data (Arnaud et al. 2001). Spectral fits were performed in the 0.5-4.0 keV band. Data below $0.5 \mathrm{keV}$ were excluded to avoid residual calibration problems in the MOS response matrices at soft energies. Data above $4 \mathrm{keV}$ were excluded because above this energy the spectra show a substantial contamination from hotter gas emitted further out in the cluster, on the same line of sight.

MOS and PN spectra are analyzed separately. As discussed in Molendi (2001), there are residual crosscalibration uncertainties between the two instruments which, as we shall see in the next sections, sometimes lead to different measurements of spectral parameters.

\section{Spectral modeling}

All spectral fitting has been performed using version 11.0.1 of the XSPEC package. All models discussed below include a multiplicative component to account for the galactic absorption on the line of sight of M 87. The column density is always fixed at a value of $1.8 \times 10^{20} \mathrm{~cm}^{-2}$, which is derived from $21 \mathrm{~cm}$ measurements (Lieu et al. 1996).
We have compared our data with three different spectral models.

Model I is a single temperature model (vmekal model in XSPEC), which allows to fit separately individual metals abundances. This model has 13 free parameters: the temperature $T$, the normalization and the abundance of $\mathrm{O}, \mathrm{Ne}, \mathrm{Na}, \mathrm{Mg}, \mathrm{Al}, \mathrm{Si}, \mathrm{S}, \mathrm{Ar}, \mathrm{Ca}, \mathrm{Fe}$ and Ni, which are all expressed in solar units.

Model II features two single temperature components (vmekal + vmekal in XSPEC) This model has two free parameters more than model I: the temperature and normalization of the second thermal component. The metal abundance of each element of the second thermal component is linked to the same parameter of the first thermal component. This model has been used in the past (e.g. Makishima et al. 2001 and refs. therein) as an alternative to cooling-flow models.

Model III includes a single temperature plus a multiphase component (vmekal + vmcflow in XSPEC). This model has two free parameters more than model I: the minimum temperature, $T_{\min }$, and the normalization of the multi-phase component. The other parameters of the multi-phase component are not free: the maximum temperature, $T_{\max }$, and the metal abundance of each element are linked respectively to the temperature, $T$, and the metal abundances of the same elements of the singlephase component. In a recent paper (Molendi \& Pizzolato 2001) we have shown that such a model fits adequately the EPIC spectra of three clusters which feature a temperature decrement in their core. The vmcflow component we use has originally been written to fit spectra from multiphase gas, i.e. gas that at a given physical radius is characterized by a distribution of temperatures rather than by a single temperature. Here we are using it to describe a different physical scenario where, at a given physical radius the gas is all at one temperature, and the multi-phase appearance of the spectrum comes from having emission from many different physical radii all within the same line of sight.

For the spectrum accumulated in the innermost region models II and III also include a power-law component to model the emission of the nucleus and of knot $\mathrm{A}$.

\section{Results}

In Fig. 1 we plot the $\Delta \chi^{2}$ between models I and II (filled circles) and models I and III (open circle) as a function of radius. The solid (dashed) horizontal line indicates the $\Delta \chi^{2}$ value for which the statistical improvement of the model II (III) fit with respect to model I is significant at the $99 \%$ level according to the F-test. The results plotted in the top panel refer to the MOS spectra while those shown in the bottom panel are for the PN spectra. From Fig. 1 we see that, for all bins and for both MOS and $\mathrm{PN}$, the improvement of models II and III with respect to model I is significant at more than the $99 \%$ level, implying that the spectra are characterized by more than just one temperature. 


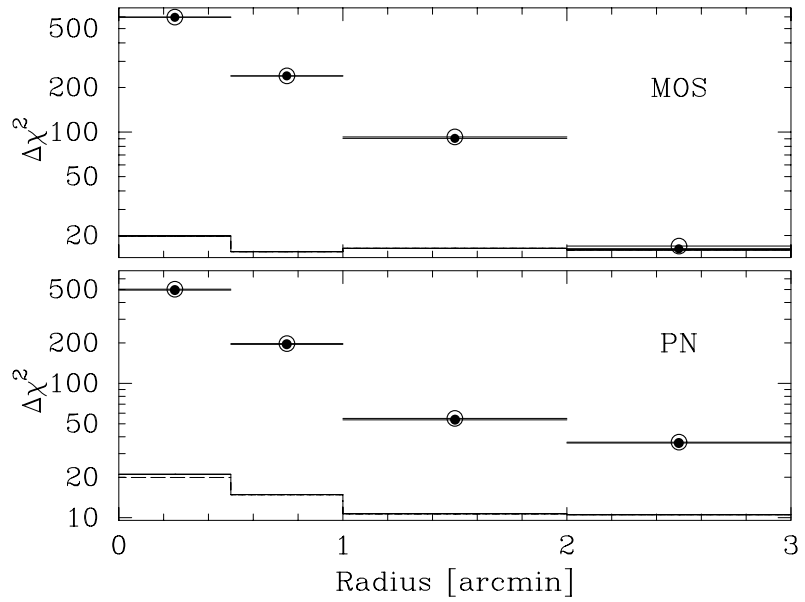

Fig. 1. $\Delta \chi^{2}$ between models I and II (filled circles), and I and III (open circles), as a function of radius. The solid (dashed) horizontal line indicates the $\Delta \chi^{2}$ value for which the statistical improvement of the model II (III) fit with respect to model I is significant at the $99 \%$ level according to the F-test. Top panel is for MOS and bottom for PN.

MOS data

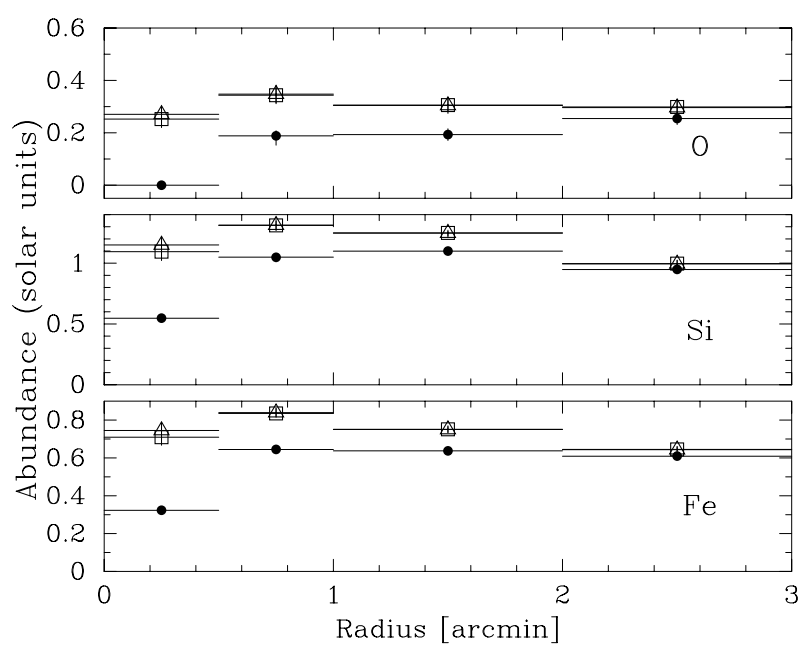

Fig. 2. MOS abundance profiles for O, Si and Fe. Uncertainties are at the $68 \%$ level for one interesting parameter $\left(\Delta \chi^{2}=1\right)$. Full symbols indicate the measurements with model I, empty squares and empty triangles those for models II and III respectively.

In the case of the innermost bin we have verified that including only the second thermal component for models II and III, provides a significantly better fit with respect to model I and that the further inclusion of the power-law component is also statistically significant. Thus for this bin we have evidence in favor of a second thermal component and of a power-law component.

In Figs. 2 and 3 we report respectively the MOS and $\mathrm{PN}$ radial abundance profiles of O (top panel), Si (middle panel) and Fe (bottom panel), while in Figs. 4 and 5 we show those for $\mathrm{Mg}$ (top panel), S (middle panel) and $\mathrm{Ar}$ (bottom panel). Full symbols indicate the measurements

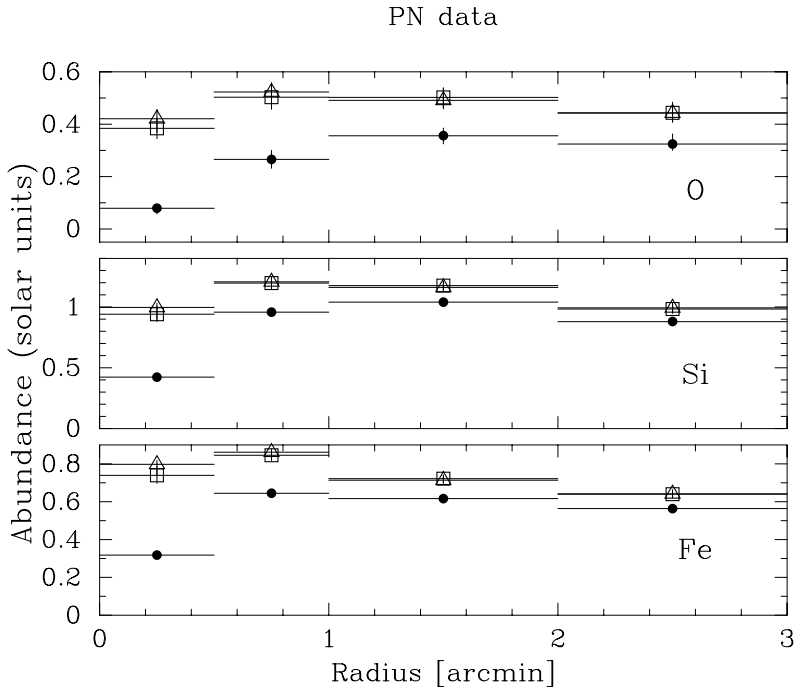

Fig. 3. PN profiles for O, Si and Fe. Symbols as in Fig. 2.

MOS data

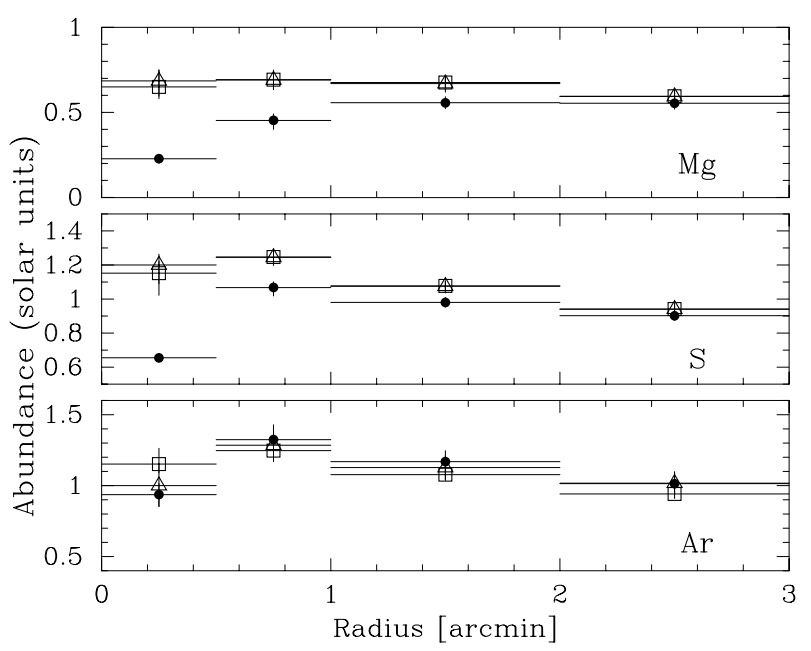

Fig. 4. MOS profiles for Mg, S and Ar. Symbols as in Fig. 2.

with model I, empty squares and triangles those with models II and III respectively.

Figures 2, 3, 4 and 5 show that, for the two innermost bins, all the metal abundances measured with model I, either with MOS or PN, are systematically smaller than those measured with models II or III. The difference between the measurements becomes larger for smaller radii as the significance of the improvement of models II and III with respect to model I (see Fig. 1) increases.

We note that, while for Si, Fe, S and Ar the measurements obtained with $\mathrm{PN}$ and MOS are consistent, for $\mathrm{O}$ and $\mathrm{Mg}$ abundances derived with the MOS are systematically higher than those derived with the $\mathrm{PN}$. The reason lies in the current cross-calibration uncertainties between the spectral response of the two instruments (Molendi 2001). However, the shape of the abundance profiles derived with models II and III is the same for MOS and PN: neither shows evidence of a dramatic metal abundance drop such as is observed with model I. 


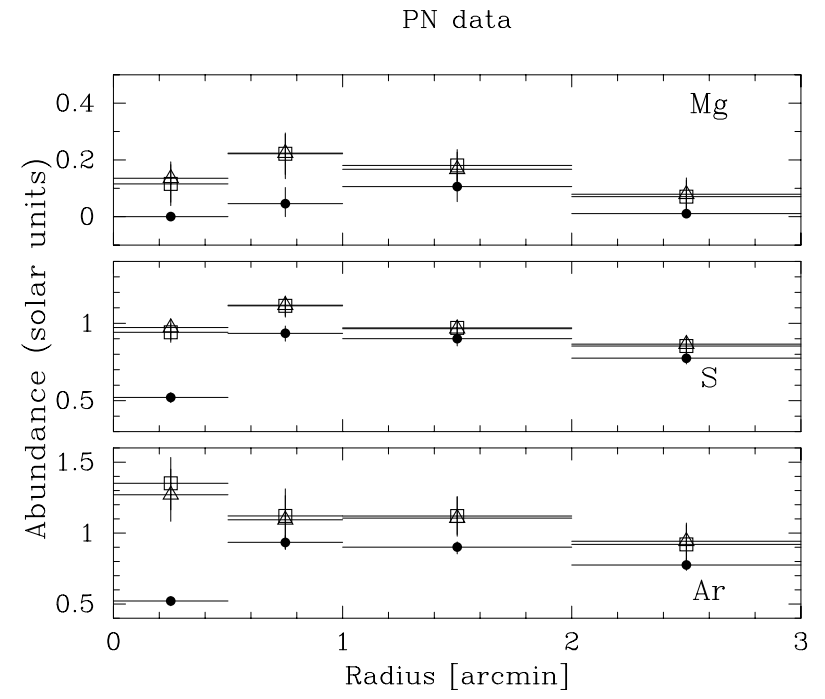

Fig. 5. PN profiles for $\mathrm{Mg}, \mathrm{S}$ and Ar. Symbols as in Fig. 2.

Clearly single temperature models substantially underestimate the metal abundance in multi-temperature spectra. This is not a new result, indeed something very similar has been observed in the ASCA spectra of galaxies and galaxy groups (e.g. Buote 2000a,b) and goes under the name of "Fe-bias". The interested reader can find a detailed discussion of the "Fe-bia" in Appendix A of Buote (2000a). The residuals of a single temperature model applied to a multi temperature spectrum around the Fe-L line complex (see Fig. 5 of Buote 2000b) are also quite illustrative.

Figure 1 tells us that models II and III behave very similarly in terms of their improvement with respect to model I. In the outermost radial bin, where we find the smallest evidence for emission from more than a single temperature, the abundances measured with models II and III do not differ substantially from those derived from model I. As we move inwards the evidence for multitemperature gas becomes stronger, the Fe-bias becomes more important, and the abundance measurements with models II and III differ more and more from those obtained with model I. In the innermost bin, the power-law emission from the nuclear sources contributes to raising the continuum with respect to the line emission thereby driving the abundances obtained with model I further down.

Since the metal abundances in the innermost region are sensitive to the normalization of the power-law component we have performed the following test to verify that this parameter is sufficiently well determined by the fitting procedure. We have accumulated a spectrum for the innermost bin excising the emission from the nucleus and knot A, using an exclusion radius of $7^{\prime \prime}$ around the sources. We recall that for such a small exclusion radius the accumulated spectrum will still be contaminated by the emission of the point-sources. We have then applied models II and III to this spectrum. We find that the best fitting values for the metallicities are in agreement with those derived by fitting the spectrum where the two sources have not been eliminated.

Close inspection of the $\mathrm{O}, \mathrm{Si}, \mathrm{Fe}$ and $\mathrm{S}$ profiles reveals a small decrement in the innermost bin, the difference between the measurement in this region and the one in region 2, which has the largest abundance, is significant at less than $2 \sigma$ for all elements in both MOS and PN with the exception of $\mathrm{Si}$ for the $\mathrm{PN}$, which is significant at about $2.7 \sigma$.

In summary, the metal abundance profiles derived by fitting single temperature models are very different from the ones that are obtained by fitting the more appropriate spectral models II or III. The former all show a dramatic abundance decrease towards the center at radii smaller than one arcmin. The latter show a flattening of the abundance profile within 1 arcmin and a hint of a decrease in the innermost bin. In the light of these results the evidence for absorption in the core of M 87 is greatly reduced. Observations at higher angular resolution with the Chandra observatory will likely settle the issue definitively.

Acknowledgements. We thank the many people who have contributed to building, calibrating and operating the EPIC instrument on-board XMM-Newton. S. Ghizzardi is thanked for useful discussions.

\section{References}

Arnaud, M., Neumann, D. M., Aghanim, N., et al. 2001, A\&A, $365, \mathrm{~L} 80$

Belsole, E., Sauvageot, J. L., Böhringer, H., et al. 2001, A\&A, 365, L188

Böhringer, H., Belsole, E., Kennea, J., et al. 2001, A\&A, 365, L181

Buote, D. A. 2000a, MNRAS, 311, 176

Buote, D. A. 2000b, ApJ, 539, 172

Fabian, A. C., Nulsen, P. E. J., \& Canizares, C. R. 1984, Nature, 310, 733

Guainazzi, M., \& Molendi, S. 1999, A\&A, 351, L19

Lieu, R., Mittaz, J. P. D., Bowyer, S., et al. 1996, ApJ, 458, L5

Makishima, H., Ezawa, H., Fukazawa, Y. 2001, PASP, in press

Mathews, W. G., Buote, \& D. A., Brighenti, F. 2001, ApJL in press [astro-ph/0101545]

Matsumoto, H., Koyama, K., Awaki, H., et al. 1996, PASJ, 48, 201

Molendi, S. 2001, Report on MOS PN cross-calibration to be presented at the Leicester EPIC calibration meeting to be held in June 2001

Nulsen, P. E. J., \& Böhringer, H. 1995, MNRAS, 274, 1093 